\title{
Characteristic scales in landslide modelling
}

\author{
E. Piegari ${ }^{1}$, R. Di Maio ${ }^{1}$, and L. Milano ${ }^{2}$ \\ ${ }^{1}$ Dipartimento di Scienze della Terra, Napoli, Italy \\ ${ }^{2}$ Dipartimento di Scienze Fisiche, Napoli, Italy
}

Received: 23 July 2008 - Revised: 17 June 2009 - Accepted: 30 June 2009 - Published: 22 July 2009

\begin{abstract}
Landslides are natural hazards occurring in response to triggers of different origins, which can act with different intensities and durations. Despite the variety of conditions that cause a landslide, the analysis of landslide inventories has shown that landslide events associated with different triggers can be characterized by the same probability distribution. We studied a cellular automaton, able to reproduce the landslide frequency-size distributions from catalogues. From the comparison between our synthetic probability distribution and the landslide area probability distribution of three landslide inventories, we estimated the typical size of a single cell of our cellular automaton model to be from $35-100 \mathrm{~m}^{2}$, which is important information if we are interested in monitoring a test area. To determine the probability of occurrence of a landslide of size $s$, we show that it is crucial to get information about the rate at which the system is approaching instability rather than the nature of the trigger. By varying such a driving rate, we find how the probability distribution changes and, in correspondence, how the size and the lifetime of the most probable events evolve. We also introduce a landslide-event magnitude scale based on the driving rate. Large values of the proposed intensity scale are related to landslide events with a fast approach to instability in a long distance of time, while small values are related to landslide events close together in time and approaching instability slowly.
\end{abstract}

\section{Introduction}

The occurrence of a landslide the size of $s$ can be quantified by the landslide size probability density function, $p(s)$, which is defined as the ratio between the number of landslides with size between $s$ and $s+\delta s$ and the total num-

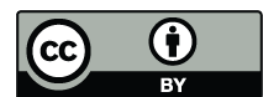

Correspondence to: E. Piegari (esterpiegari@gmail.com) ber of landslides in an inventory and divided by $\delta s$. The analysis of landslide inventory maps shows that $p(s)$ exhibits two regimes: an increasing behaviour for small landslides and a power-law scaling, with a negative scaling exponent, for large landslides. Several investigators have recently proposed different frequency-size probability distributions to describe the landslide size statistics. Stark and Hovius (2001) address the use of a modified Pareto (power-law) distribution, characterized by a double (positive and negative) power scaling, to describe historical inventories, i.e. inventories that include events occurred over time. Malamud et al. (2004a), instead, analyse fresh inventories, i.e. inventories accomplished shortly after a landslide event, and propose an inverse-gamma probability distribution, characterized by a power-law decay for medium and large landslides and an exponential rollover for small landslides. Both these approaches retrieve the characteristic distribution a posteriori as the best fit of data sets of specific events.

Conversely, we propose a cellular automaton model (CA) aimed at reproducing the landslide size distribution a priori by means of some characteristic parameters (Piegari et al., 2006a). In this way, our attention is mainly focused on the determination of the key ingredients that lead a landslide of size $s$ to have a probability of occurrence $p(s)$, rather than finding a general frequency-size distribution. We find that the behaviour of $p(s)$ strongly depends on the rate $v$ at which the system approaches instability, changing from power-law to non power-law behaviour.

In the following, we analyse the behaviour of both the probability density function (pdf) of having a landslide of size $s, p(s)$, and the pdf of having a landslide of lifetime $t_{L}$, $p\left(t_{L}\right)$, in the limit of vanishing $v$ and for finite values of $v$. We discuss the shift of the maximum of $p(s)$ with the increasing of $v$ in connection with the behaviour of $p\left(t_{L}\right)$, by indicating a change in the dynamics of the landslide process. Moreover, we compare the synthetic $p(s)$ of our CA with the real landslide area pdf of three landslide events providing an estimate for the units of measure of the CA cells. We

Published by Copernicus Publications on behalf of the European Geosciences Union and the American Geophysical Union. 


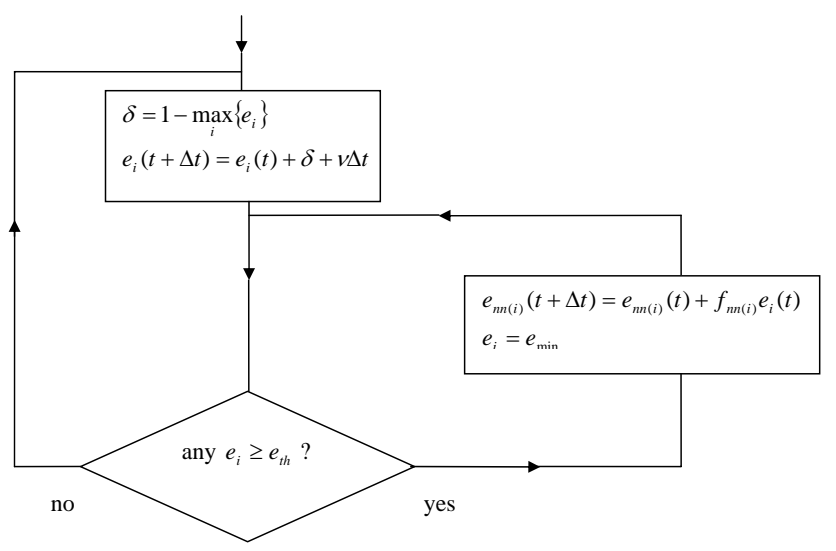

Fig. 1. Flow chart of the cellular automaton model. The parameter $v$ represents the driving rate, i.e. the rate at which instability is approached, while the $f_{n n}$ coefficients quantify how instability is transmitted from a cell to its nearest cells. The subindex $n n(i)$ stands for all nearest neighbours of the cell $i$ (i.e., $n n(i)=$ up, down, left, right).

also propose an intensity scale related to the rate at which the system approaches instability.

\section{The Cellular Automaton model (CA)}

In order to simulate a landslide event, we partition a natural slope by means of a two-dimensional square grid of $L \times L$ cells. Each cell $i$ of the grid represents an area characterized by a local value of the factor of safety, $F S_{i}$. In slope stability analysis, the factor of safety, $F S$, is defined in terms of the ratio of the maximum shear strength $\tau_{\max }$, given by the empirical Mohr-Coulomb expression, to the disturbing shear stress $\tau$ (Fredlund and Rahardjo, 1993). If $F S>1$, resisting forces exceed driving forces and the slope remains stable. Slope failure starts when the safety factor reaches the critical value $F S=1$. To simplify numerical simulation, we consider the inverse of the local factor of safety, $e_{i}=1 / F S_{i}$ as the dynamical variable of our model. We start from a random initial stable configuration, i.e. we attribute to each cell a uniformly distributed random value of $e_{i}$ with $0<e_{i}<1 \forall i$. The dynamics of the CA model is defined by the following two rules:

$\left\{\begin{array}{l}e_{i}(t+\Delta t)=e_{i}(t)+\delta+v \Delta t \\ \delta=e_{t h}-\max \left\{e_{i}\right\}\end{array}\right.$

$e_{i} \geq e_{t h} \rightarrow\left\{\begin{array}{l}e_{n n(i)}(t+\Delta t)=e_{n n(i)}(t)+f_{n n(i)} e_{i}(t) \\ e_{i}=e_{\min }\end{array}\right.$

where $n n(i)$ denotes the four neighbour sites of the overcritical site $i$ (i.e. $n n(i)=$ up, down, left, right). To aid the reader, a list of variables used in the text is given in Table 1.

The first rule, Eq. (1), is an overall driving that provides an increase of $e_{i}$ at the same rate approaching the system to the instability threshold, $e_{t h}=1$. We set to 1 the elementary time step $\Delta t$. The parameter $v$ controls the rate at which all sites are driven towards instability, while the difference $\delta$ between the instability threshold $e_{t h}$ and the largest $e_{i}$ value is just a technical expedient to treat the limit of vanishing driving rate $v$. In this case, only the site (or very few sites) with $e_{i}=\max \left\{e_{i}\right\}$ reaches the instability threshold first. The second rule, Eq. (2), is a relaxation rule: when a cell becomes unstable (i.e. $e_{i} \geq e_{t h}$ ), it affects, via a chain reaction, the stability of the neighbour cells, as a fraction $f_{n n(i)}$ of $e_{i}$ toppling on $n n(i)$. After a failure, we set $e_{i}=e_{\min }$ with $e_{\min }=10^{-6}$. We mention that any other finite level would work (Jensen, 1998) and our numerical results do not change up to values of $e_{\min }=10^{-1}$. During each iteration of Eq. (2), an amount of $e_{i}$ is lost from the system, that is the difference between $e_{i}$ and the amount $f_{n n} e_{i}$ added to each of the four neighbour sites. Only if $\sum_{n n} f_{n n}=1$, the algorithm conserves the dynamical variable of the system.

Thus, another crucial parameter of the CA is the quantity $C=\sum_{n n} f_{n n}$ that fixes the degree of conservation of the system. Contrary to most numerical models for avalanches (Hergarten and Neugebauer, 2000; Hergarten, 2003), we consider a nonconservative case, $C<1$, to describe landslide processes, since many complex dissipative phenomena (Fredlund and Rahardjo, 1993) can contribute to stress transfer processes. The dependence of the CA pdf on $C$ is shown in Piegari et al. (2006a), while the dependence on the coefficients $f_{n n}$ has been studied in detail in Piegari et al. (2006b). In particular, we have found that the pdf of having a landslide with $s$ cells involved, $p(s)$, has a negative power-law exponent that is a decreasing function of $C$, while it is not significantly affected by the values of the coefficients $f_{n n}$ in the range of values that supply power-law distributions.

The algorithm of the proposed CA is illustrated by the flow chart shown in Fig. 1: the inner loop of the chart describes the stages of an individual landslide, while the outer loop describes a sequence of landslide events. We study the spatial and temporal pdf of the CA once the system has attained a stationary state in its dynamics, i.e. the mean value of the dynamical variable, $e_{i}$, on the grid sites fluctuates between an average value.

We notice that the values of the transfer coefficients, $f_{n n}$, may be different for each site, by opening the possibility to treat with relaxation processes that take into account the topography of a specific slope (Piegari et al., 2009).

\section{Probability density functions of landslide events of definite size and lifetime}

In this section, we discuss the behaviour of both the probability density functions, pdf, of having a landslide of size $s$, $p(s)$, and of having a landslide of lifetime $t_{L}, p\left(t_{L}\right)$, in the limit of vanishing $v$ and for finite values of $v$. In the proposed CA model, the size $s$ is defined as the number of cells 
Table 1. Variables used in the text.

\begin{tabular}{|c|c|c|}
\hline Variable & Description & $\begin{array}{l}\text { Equation/Section } \\
\text { introduced }\end{array}$ \\
\hline$\alpha$ & Critical index that controls how the finite-size cutoff scales with the system size. & Eq. (4) \\
\hline$\beta$ & Critical index related to the renormalization of the probability density function & Eq. 4 \\
\hline$v$ & Parameter that controls the rate at which instability is approached. & Eq. (1) \\
\hline$a$ & Coefficient in $s^{*} \times a=A^{*}$ & Sect. 3.2 \\
\hline$A$ & Area of landslide. & Sect. 3.2 \\
\hline$A^{*}$ & $\begin{array}{l}\text { Landslide area corresponding to the maximum of the probability density function of having a } \\
\text { landslide of area } A \text {. }\end{array}$ & Sect. 3.2 \\
\hline$C$ & Level of conservation of the system $C=\sum_{n n} f_{n n}$ & Sect. 2 \\
\hline$e_{i}$ & Inverse of the local value of the safety factor for the cell $i$. & Sect. 2 \\
\hline$e_{t h}$ & Instability threshold. & Eq. (2) \\
\hline$e_{\min }$ & Relaxation threshold. & Eq. (2) \\
\hline$f_{n n}$ & Instability transfer coefficients. & Eq. (2) \\
\hline$F S$ & Safety factor. & Sect. 2 \\
\hline$F S_{i}$ & Local value of the safety factor for the cell $i$. & Sect. 2 \\
\hline$g$ & Scaling function of the ratio $s / L^{\alpha}$. & Eq. (4) \\
\hline$L$ & Square root of the total number of cells. & Sect. 2 \\
\hline$M_{L}$ & Magnitude of a landslide event, with $M_{L}=\log _{10}\left(\frac{T_{L}}{v^{-1}}\right)$ & Eq. (7) \\
\hline$n n(i)$ & Neighbour sites of the overcritical site $i$ (up, down, left, right). & Eq. (2) \\
\hline$p(s)$ & Probability density function of having a landslide of size $s$. & Sect. 1 \\
\hline$p\left(t_{L}\right)$ & Probability density function of having a landslide of lifetime $t_{L}$. & Sect. 3 \\
\hline$s$ & Total number of cells that reach the instability threshold in a chain relaxation process. & Sect. 3 \\
\hline$s^{*}$ & Value of $s$ corresponding to the maximum of $p(s)$ & Sect. 3.2 \\
\hline$<s>$ & Mean value of $s$ & Eq. (5) \\
\hline$t_{L}$ & Landslide lifetime: number of loops up to when an unstable site exists. & Sect. 3 \\
\hline$<t_{L}>$ & Mean value of $t_{L}$ & Eq. (6) \\
\hline$T_{L}$ & Time interval between two landslide events in a specific area. & Sect. 4 \\
\hline
\end{tabular}

that reach the instability threshold in a chain relaxation process, and, therefore, $s$ can be considered a proxy for the area of a real landslide. The lifetime of an avalanche event, $t_{L}$, is defined by the number of avalanching loops up to when an unstable site exists, and, therefore, $t_{L}$ can be considered a proxy for the lifetime of a landslide.

\subsection{The limit of vanishing driving rate}

In the limit $v=0$, the model provides results similar to those of the most studied cellular automaton for earthquakes, i.e. the Olami-Feder-Christensen model (OFC) (Olami et al., 1992). In such a limit, no scales characterize the model: the connections between the size $s$ and the linear dimension of the system $L$, as well as the connections between the lifetime $t_{L}$ and $L$, are described by a scale-independent relation, i.e. by a power-law.

Let $p(s, L)$ be the probability density of having a landslide of size $s$ in a system of linear size $L$. If $p(s, L)$ is a power law, we can define the power law exponent, $B$ :

$p(s, L) \approx s^{-(1+B)}$
The scaling properties of the system are investigated by finite-size-scaling analysis (Privman, 1990), i.e. it is assumed that the pdf scales with the system size as:

$p(s, L) \approx L^{-\beta} \cdot g\left(\frac{s}{L^{\alpha}}\right)$,

where $g$ is a so-called universal scaling function and $\beta$ and $\alpha$ are known as critical indices that describe the scaling properties of the system: $\beta$ is related to the renormalization of the distribution function, while $\alpha$ controls how the finite-size cutoff scales with the system size. If $p(s, L)$ is a power law, then $1+B=\frac{\beta}{\alpha}$ (Christensen and Olami, 1992).

We calculate the indices $B, \alpha$ and $\beta$ by considering statistics of over $10^{9}$ events per run and fix the values of the anisotropic transfer coefficients $f_{n n}$, which we set equal to: $f_{\text {up }}=0.1, f_{\text {down }}=0.3, f_{\text {left }}=f_{\text {right }}=0.2$. This choice for the $f_{n n}$ values implies that $C=\sum_{n n} f_{n n}=0.8$. The results are shown in Figs. 2 and 3. In Fig. 2, we plot the product $L^{\beta} p(s, L)$ as a function of the ratio $s / L^{\alpha}$ for two different linear size $L$, in a $\log$ - $\log$ scale. We find the values of the critical indices $\alpha=1.43$ and $\beta=2.34$, as the values for which the two curves are overlapped. The value of the exponent of 


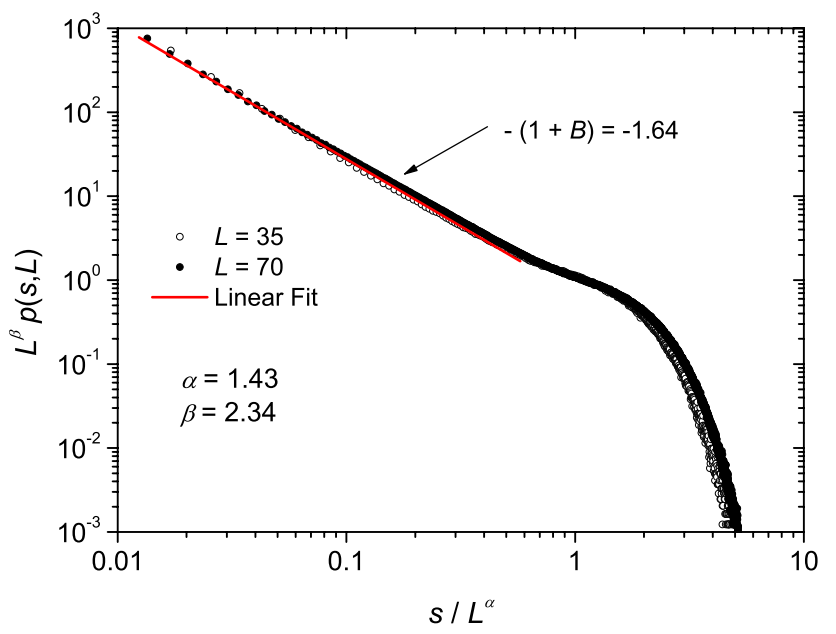

Fig. 2. $L^{\beta} p(s, L)$ vs. $s / L^{\alpha}$ for two values of the linear system sizes, $L=35$ and $L=70$. The values of the finite scaling exponents that determine the overlapping of the curves are $\alpha=1.43$ and $\beta=2.34$. Variables on axes are unitless.

the negative power-law, $-(1+B)$, is obtained from the linear fit of the curves, by neglecting the points relative to the cut-off due to the finite size of the CA. We notice that the values obtained for $\alpha$ and $\beta$ in Fig. 2 differ from those provided by the OFC model for the same level of conservation $C=0.8$ (Christensen and Olami, 1992). In contrast to the anisotropy investigated in the OFC model, the anisotropy introduced in our modelling breaks the up-down symmetry. It has showed that such a symmetry breaking can change the universality class in driven non-equilibrium systems (Pruessner and Jensen, 2002).

In Fig. 3, we report the product $L^{\beta} p\left(t_{L}, L\right)$ as a function of the ratio $t_{L} / L^{\alpha}$ for the same values of $L$ shown in Fig. 2. We find the values of the critical indices $\alpha=1.03$ and $\beta=1.70$ as the values for which the curves, corresponding to different values of the linear size, are overlapped. The slope of the linear fit (solid line) gives the value of the exponent of the negative power-law, $-(1+B)=-1.65$.

A landslide lifetime distribution in nature would be a function that records the number of landslides with time duration between $t_{L}$ and $t+\delta t_{L}$, divided by the total number of landslides in a complete landslide inventory and divided by $\delta t_{L}$. The authors are not aware of landslide lifetime distributions from empirical data and suggest an inventory analysis as a further testing of the model.

\subsection{Finite values of the driving rate}

Let us focus, now, on the features of the model when finite values of the driving rate $v$ are taken into account. As discussed in previous papers (Piegari et al., 2006a, b), when the driving rate $v$ increases, the landslide size pdf develops a maximum that shifts towards larger sizes. For small land-

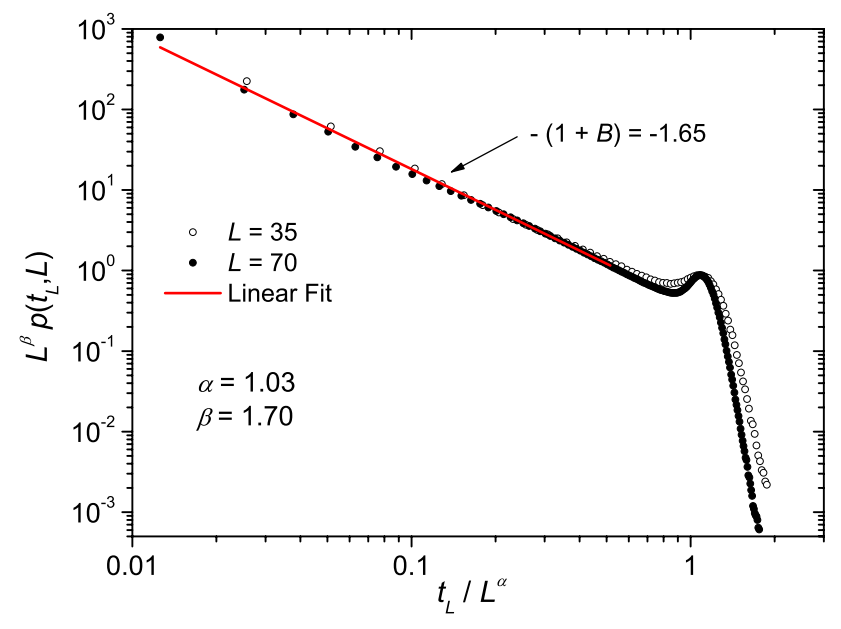

Fig. 3. $L^{\beta} p\left(t_{L}, L\right)$ vs. $t_{L} / L^{\alpha}$ for two values of the linear system sizes, $L=35$ and $L=70$. The values of the finite scaling exponents that determine the overlapping of the curves are $\alpha=1.03$ and $\beta=1.70$. Variables on axes are unitless.

slides, we find a positive power-law, while for medium and large landslides the CA model provides a negative powerlaw. We studied the evolution of the positive and negative power-law exponents with the drive $v$ in Piegari et al. (2006b) and we found that the slope coefficient of the negative powerlaw is not a monotonic function of $v$, while for the positive power-law the slope coefficient is an increasing function of $v$.

We compare our synthetic pdf with those coming from three landslide inventories and the inverse gamma distribution proposed by Malamud et al. (2004a). Figure 4 shows this comparison considering the synthetic distributions obtained for three sets of the parameters $v$ and $C(\nu=0.003$ and $C=0.5 ; \nu=0.003$ and $C=0.4 ; \nu=0.005$ and $C=0.4$ ). In the figure, our distributions are plotted as a function of the landslide area, which is a measure of the size of the cells involved in the avalanche event. We convert the landslide size $s$ in the corresponding area by multiplying the unitary size of a cell for an area factor $a$. Such a factor has been obtained by imposing that $s^{*} \times a=A^{*}$, where $s^{*}$ is the size corresponding to the maximum of a synthetic pdf and $A^{*}$ is the area corresponding to the maximum of the pdf from real data. As it can be seen, the agreement between the synthetic and the real curves is quite good. By varying the value of the conservation level $C$, while $v$ is kept fixed, we find that the slope of the negative power-law changes smoothly up to include all the real data. If $C$ is kept fixed, small variations of $v$ take into account the tiny differences in the slope of the rollover for small landslides. In Fig. 4, it is also shown the inverse gamma distribution (black solid line) proposed by Malamud et al. (2004a). As observed, the agreement between such a curve and our synthetic distribution is also quite good, with the difference that the inverse gamma distribution is found a 
posteriori as the best fit of the real data and it is unique for all the landslide events, while our pdf is generated a priori from the proposed CA model and depends on the values of some key parameters. We point out that, for very large areas, the deviation of the synthetic pdf from the real ones is an effect of the finite size of the system that produces the cut-off. If grids with larger $L$ are considered, such a cut-off shifts to larger areas.

Moreover, it is worth noticing that the comparison with the real data, shown in Fig. 4, allows us to determine the typical size of a single cell of our grid, which is crucial information if we are interested in monitoring a test area. In detail, we find that the area of a single cell $a$ ranges from $35 \mathrm{~m}^{2}$ to $60 \mathrm{~m}^{2}$ in the case $C=0.4$, and it is of the order of $100 \mathrm{~m}^{2}$ in the case $C=0.5$.

As discussed in Malamud et al. (2004a), even if the three landslide inventories of Fig. 4 correspond to three different triggering mechanisms, they seem to obey the same pdf. From the analysis of our CA, we realize that triggers of different origins can affect the stability of a slope with the same rate of approaching instability, i.e. with the same $v$. Such an observation suggests that to characterize the probability of occurrence of landslide events in a specific area, it is crucial to get information about such a rate $v$ that controls the time evolution of the slope stability, rather than the nature of the triggering mechanism.

It is worth pointing out that varying $v$ in the CA model means changing the rate at which the system reaches instability. As fresh inventories come from a mapping carried out shortly after a triggered landslide event, it can be described by a synthetic pdf corresponding to a fixed value of $v$, since it seems reasonable to assume that the landslides are essentially triggered with the same rate to approach instability. On the contrary, an historical inventory, which is the sum of many landslide events over time, very likely includes landslides corresponding to different rates of their approach to instability, and, therefore, the relative pdf could be considered a weighted average of distributions with different $v$.

As discussed in detail below, we find that large values of $v$ cause the simultaneous instability of large areas. This feature of the model could explain the shift to the right of the pdf maximum that describes historical inventories: as time moves on, the evidence of smaller landslides is more likely to be lost, and the historical inventories include just the most catastrophic events corresponding to larger values of $v$. As a result, the rollover of the pdf moves to the right. Finally, we notice that our explanation for the shift of the maximum rollover typical of historical inventories is compatible with that proposed by Malamud et al. (2004b). They attribute the shift of the pdf maximum to the incompleteness of the historical inventories caused by the erosion: when going to historical data sets, the evidence for the existence of many smaller and medium landslides is lost due to wasting processes over time and, therefore, an historical inventory turns out to contain only the largest landslides (Malamud et al., 2004b).

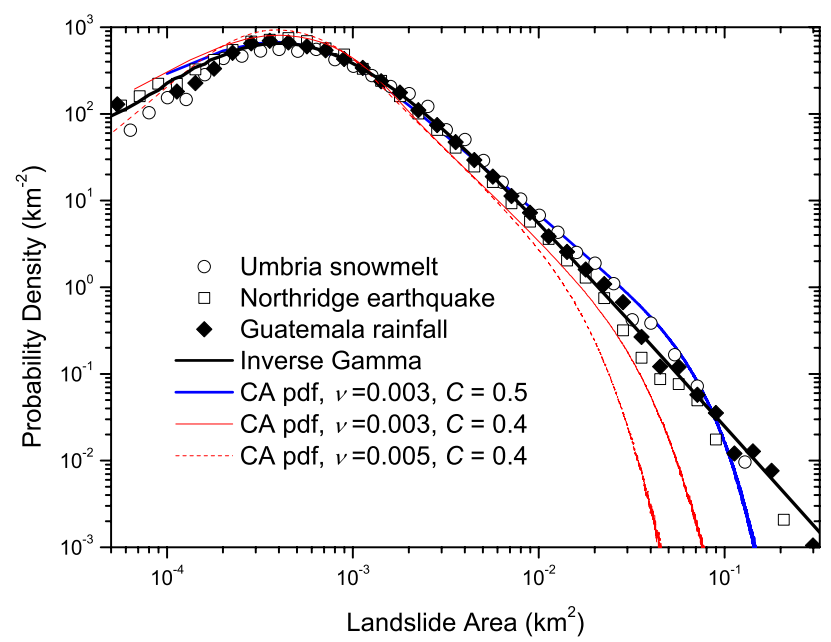

Fig. 4. Landslide probability densities as a function of the area. Symbols are used for the three landslide inventories from Malamud et al (2004a). The black solid line corresponds to the inverse gamma distribution proposed by Malamud et al. (2004a). The colour lines refer to our synthetic curves for different values of the driving rate $v$ and the conservation level $C$, as shown in the legend.

\subsection{Analysis of the landslide dynamics with the driving rate}

To better understand the features of the model by varying the driving rate $v$, we study the evolution of $p(s)$ in connection with the behaviour of $p\left(t_{L}\right)$ with increasing $v$.

In Figs. 5 and 6, we plot, respectively, $p(s)$ and $p\left(t_{L}\right)$ for different values of $v$ and for a smaller system linear size than that of Fig. 4, to reduce the computation time. In the limit of vanishing driving rate, it has been shown that the distribution function $p(s)$ describes avalanche events that are essentially compact clusters of sites (Pietronero and Schneider, 1991; Piegari et al., 2006a). In such a case, the instability starts from a single cell and, then, propagates to neighbour cells generating avalanche events of sizes that are power-law distributed. Increasing the value of the driving rate $v$ means to enhance the chances to generate the simultaneous instability of more than one cell. Thus, the larger $v$ is, the larger the number of relaxation chain processes that may originate in the system. For this reason, we realize that the size $s^{*}$, corresponding to the maximum of $p(s)$ for finite values of $v$, is essentially an estimate of the total number of cells that, at the start, simultaneously reach instability. Interestingly enough, we find that (see Fig. 5), for a given range of very low $v$ values, the maximum of $p(s)$ is followed by a powerlaw regime that resembles the landslide frequency-size distributions from catalogues. As it can be seen in Fig. 5, a further increase of $v$ causes a crossover to a clearly different regime where power-laws are no longer apparent and a bell-shaped distribution emerges, whose peak shifts towards larger sizes and shrinks up. In this limit, an avalanche event 


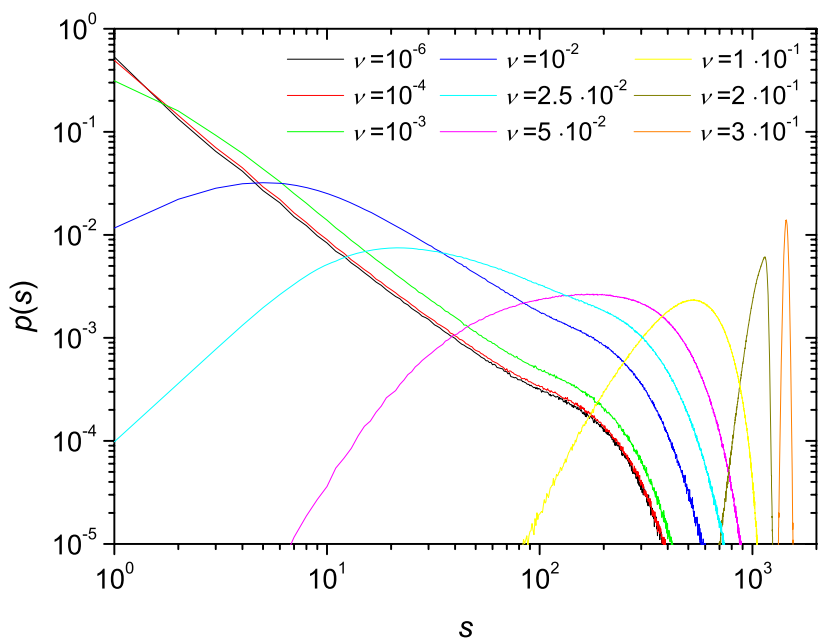

Fig. 5. Evolution of the probability density $p(s)$ of landslides of size $s$ with the driving rate $v$, in a log-log scale. A crossover from power-law to non power-law behaviour is apparent and a bellshaped (Gaussian) distribution emerges, whose peak shifts towards larger sizes with increasing $v$. Variables on axes are unitless.

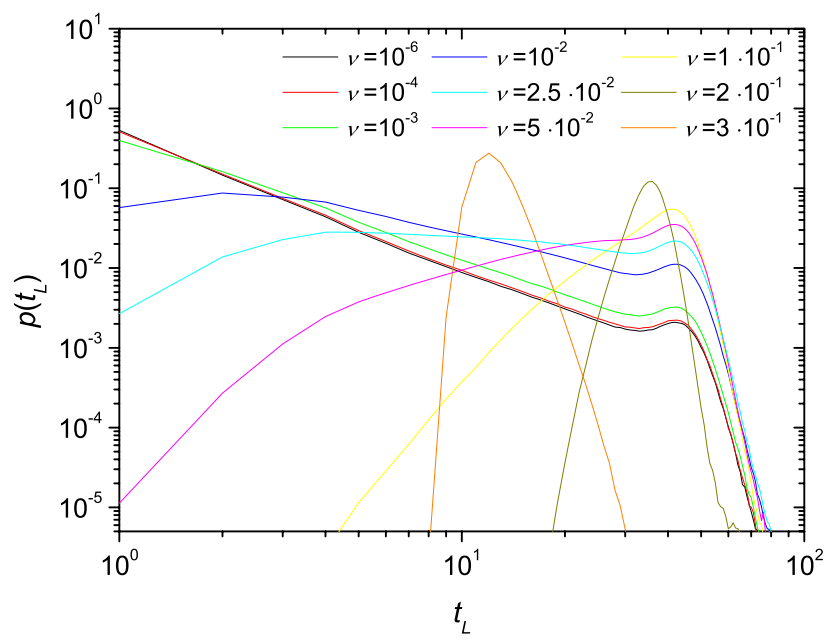

Fig. 6. Evolution of the probability density $p\left(t_{L}\right)$ of landslides of lifetime $t_{L}$ with the driving rate $v$, in a $\log$ - $\log$ scale. A crossover from power-law to non power-law is apparent and a bell-shaped distribution emerges, whose peak shifts towards smaller sizes with increasing $v$. Variables on axes are unitless.

occurs because a very large number of cells instantaneously reach instability, instead of resulting from a propagation of a local instability.

Such features of the model, which demonstrate a change in the model dynamics with increasing $v$, can also be observed in the behaviour of the landslide lifetime distribution $p\left(t_{L}\right)$. As seen in Fig. 6, the probability density function $p\left(t_{L}\right)$ has a non-trivial behaviour with $v$. In the limit of vanishing $v$, it shows a power-law scaling that is well evident for the same

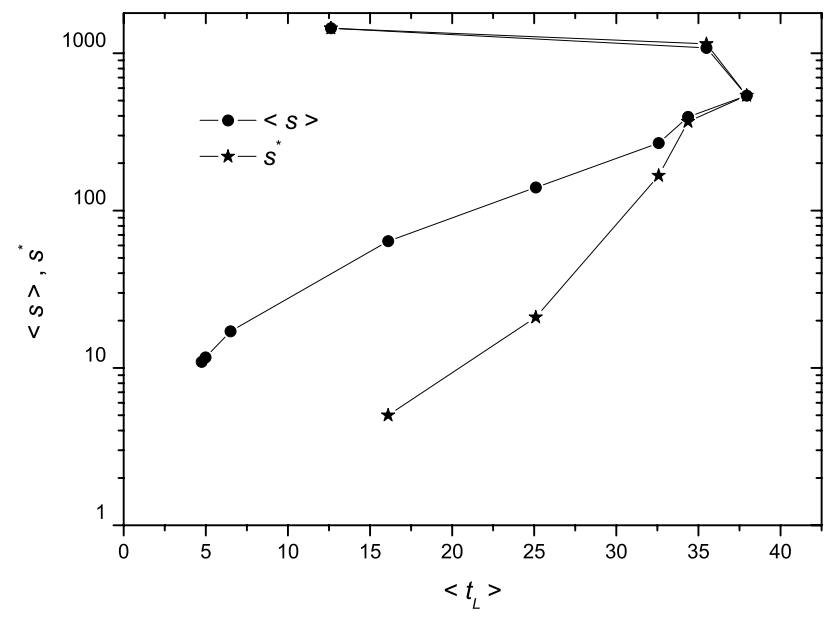

Fig. 7. The maximum of $p(s), s^{*}$, and the landslide mean size, $<s>$, as functions of the landslide mean lifetime $\left\langle t_{L}>\right.$ for different values of the driving rate $v$, as shown in Figs. 5 and 6 . Variables on axes are unitless.

values of $v$ for which $p(s)$ shows power-law scaling. Then, $p\left(t_{L}\right)$ starts to bend for an increasing interval of $v$ values and becomes, for very large $v$, a bell-shaped distribution, whose peak shifts towards smaller lifetimes. The behaviour of $p\left(t_{L}\right)$ at varying values of $v$ reveals a continuous modification with $v$ of the dynamical processes causing landslides. An increase of $v$ causes an enhancement of the number of cells that initially reach instability, $s^{*}$. Initially, the larger $s^{*}$ is, the larger the lifetime of the more likely events, i.e. the larger is the number of cells involved in avalanching loops that generate chain relaxation processes. Then, we find that for a further increase of $v$, the maximum of $p\left(t_{L}\right)$ moves towards lower values of $t_{L}$. To better explain this feature, we calculate for each examined value of $v$ the predicted landslide mean size $<s>$ and the predicted landslide mean lifetime $<t_{L}>$ respectively defined as:

$$
\begin{aligned}
& <s>=\int s p(s) d s \\
& <t_{L}>=\int t_{L} p\left(t_{L}\right) d t_{L} .
\end{aligned}
$$

In Fig. 7, we report both $s^{*}$ and $<s>$ as a function of $\left\langle t_{L}>\right.$ for the values of $v$ considered in Figs. 5 and 6. As it can be seen, both $s^{*}$ and $\left\langle s>\right.$ are increasing functions of $\left\langle t_{L}>\right.$ up to $v \approx 10^{-1}$. A further increase of $v$ causes larger events characterized by smaller lifetimes. We realize that in this regime the domino processes, which characterize the landslide events for small and medium $v$, are no more effective in causing avalanching processes and the instability is reached simply because a very large number of cells almost instantaneously reach the critical threshold. Thus, it is reasonable to consider that a very large $v$ describes the effects of 
critical strengths of triggering mechanisms that cause landslide events involving very large areas in a short time.

Finally, we notice that from our study it follows that the value of the parameter $v$ is strictly connected to the maximum rollover $s^{*}$. The analysis of landslide inventories has shown that landslide events associated with different triggers can be characterized by the same probability distribution, and, therefore, by the same $s^{*}$. Since in the proposed CA model $s^{*}$ is essentially an estimate of the most likely number of cells that simultaneously reach instability at the start of the landslide processes, we argue that such a quantity for a triggered event inventory is not a mere artefact of limited mapping resolution (Guzzetti et al., 2002; Malamud et al., 2004a, b) and might represent a fixed thickness of an unconsolidated layer situated at the upper part of a slope as suggested by Katz and Aharonov (2006). It is also worth noticing that in the CA model the absence of a rollover in the frequency-size probability distribution is found in the limit of very small values of $v$, which describe chain reactions that originate from a single cell and are power-law distributed. Thus, in our approach the typical power-law behaviour of rock-fall size distributions, which do not show a rollover, can be explained as a result of a specific mechanism of instability propagation rather than the absence of an unconsolidated layer (Katz and Aharonov, 2006).

\section{A proposal for a landslide-event intensity scale}

Whereas for earthquakes well-known magnitude scales have been identified, which help the general understanding of the implications of an earthquake, for landslide events this issue is still open. Keefer (1984) has proposed a magnitude scale to quantify the number of landslides in earthquake-triggered landslide events. Recently, Malamud et al. (2004a) have suggested a landslide-event magnitude scale independent on the triggering mechanism and based on the logarithm to the base 10 of the total number of landslides associated with an event. Alternatively to these approaches, which relate the landslide magnitude scale to the mapped mass movements, i.e. to the visible effects of the triggering mechanisms, we attempt to relate the landslide event magnitude scale to the strength of the trigger. We introduce, in fact, the intensity scale $M_{L}$ in terms of the rapidity $v$ of the system to reach instability:

$M_{L}=A+\log _{10} v$

where $A$ is a constant introduced to get dimensionless the scale $M_{L}$. We define $A=\log _{10} T_{L}$, with $T_{L}$ the time interval between two landslide events in a specific area. Such a choice is motivated by the following consideration. As discussed above, $v$ is the rapidity at which the system reaches instability, therefore $v^{-1}$ is an estimate of the time needed to build up the critical stress in the slope. In an actual slope, we realize that the random distribution of the safety factor values, which captures the heterogeneity of the soil, fluctuate around a mean value as response to the interaction with climate and/or external perturbations. Only if the action of a trigger causes a monotonic change (a decrease) of the FS values, the whole system moves towards the instability in the time $v^{-1}$. It follows that the characteristic time $v^{-1}$ is always smaller than $T_{L}$ and, therefore, $M_{L}=\log _{10}\left(\frac{T_{L}}{v^{-1}}\right)$ is positively defined. We point out that the limit case $\nu=0$ is not attainable in actual landslide processes (where physical interactions are controlled by finite characteristic times), whereas very small finite values of $v$ describe mass movements characterized by only a chain process, typical of rockfalls, which are not considered in this work.

From Eq. (7), it follows that large values of the proposed intensity scale are related to events with long time distances and short build-up stress times $v^{-1}$ (i.e. with fast approaching of instability), while small values of $m_{L}$ correspond to events close together in time and approaching instability slowly. Obviously, the quantity $m$ can be measured only if the system is time monitored, in such a way it would be possible to appreciate the temporal variations of the safety factor related to $v$. This is, surely, not an easy task, like the estimation of the safety factor, but it is not an unattainable task.

Recently, in fact, Juanico et al. (2008) have demonstrated experimentally our theoretical result (Piegari et al., 2006a) concerning the existence of a crossover from power-law to non power-law statistics with the driving rate. They examine avalanche statistics of rain- and vibration-driven granular slides in sand mounds and give an estimate of $v$ in terms of experimental parameters. Moreover, to evaluate the safety factor for shallow landslide, it is often the approximation of an infinite-slope used for the expression of FS (Sidle, 1995; Montgomery and Dietrich, 1994; Dietrich et al., 1995; Wu and Sidle, 1995). By using this analytical expression for the safety factor, its time variation, i.e. $v$, has been evaluated by Iverson (2000) in relation to a pressure-head response function, which depends on the intensity and duration of the trigger (rainfall). Another approach for the estimation of the safety factor, and its temporal variation, has been recently proposed also by the authors (Piegari et al., 2009). In particular, we suggest relating the local slope stability of pyroclastic covers to the local slope angle and the mean electrical resistivity value measured in a cell of the grid test area. In such a case, the value of $v$ is related to variations of the water content of the pyroclastic cover, which can be monitored through the changes of the electrical resistivity values.

Let us proceed now in giving an estimation of the landslide magnitude values based on the proposed intensity scale (Eq. 7). From a previous analysis of the model in the range of parameter values $v \in\left[10^{-4}, 10^{-2}\right]$ and $C=0.4$ (Piegari et al., $2006 a, b)$, we have found that the frequency-size distribution exhibits inverse power-law behaviours with exponents similar to those of the pdf from real data. In this case, if we put $T_{L}$ equal to the largest value of $v^{-1}$ for which the distribution 


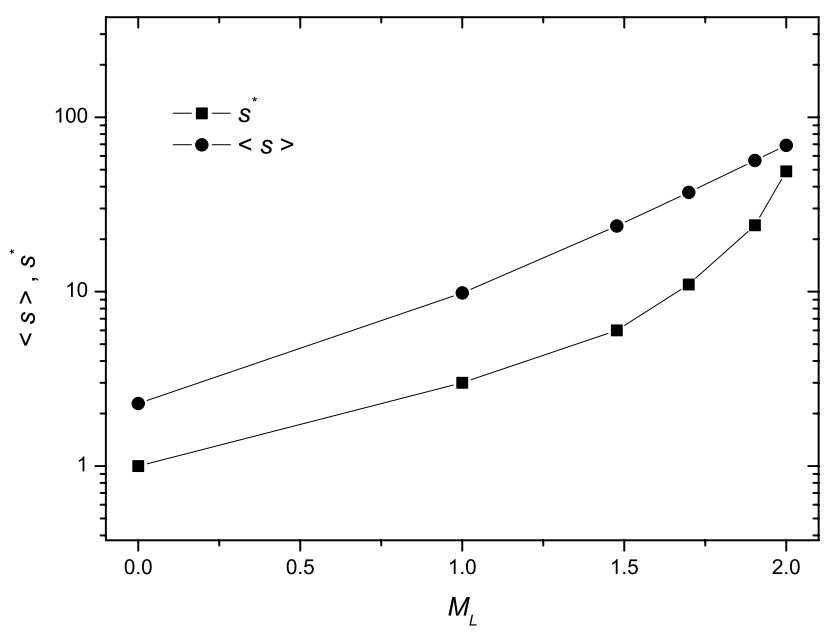

Fig. 8. The most probable event size $s^{*}$ and the predicted mean event size $<s>$ as functions of the magnitude $M_{L}$ in the range [0, 2]. The sizes are given on logarithmic axis and $M_{L}$ on linear axis. Variables on axes are unitless.

resembles a maximum, i.e. $T_{L}=10^{4}$, we obtain a magnitude scale for landslides that varies in the range $0<M_{L}<2$. We remind the reader that in our theoretical approach, the characteristic times $v^{-1}$ and $T_{L}$ are unitless and they can be measured only if the model is applied to a specific monitored survey area.

Finally, in Fig. 8, we show the most likely event size $s^{*}$ - corresponding to the maximum of $p(s)$ - and the predicted mean event size $<s>$, as functions of the magnitude $M_{L}$. The value of $s^{*}$ and $<s>$ are obtained from the distributions that resemble the experimental ones shown in the previous works (Piegari et al., 2006a, b). Conversely to the approach that proposes a general landslide probability distribution (Malamud et al., 2004a), we find that both the characteristic sizes $s^{*}$ and $<s>$ increase with the magnitude scale. The dependence of $\left\langle s>\right.$ on $M_{L}$ is essentially linear in a log-linear scale. Instead, the enhancement of $s^{*}$ is such that $s * \rightarrow<s>$ in the limit of very large values of $M_{L}$ (i.e., $M_{L}>2$ ). This evidence could be justified considering that for such values of $M_{L}$ the frequency-size distribution becomes a good approximation of a Gaussian distribution, where the most probable event coincides with the mean event.

\section{Conclusions}

Summarizing, we have found that the rate of approaching instability, $v$, is a crucial ingredient to quantify the probability of the occurrence of a landslide of size $s$. We have performed a statistical analysis of the proposed CA model by varying $v$ in an uniform way. From the analysis of the spatial and temporal probability distributions, we have found that landslide events triggered by different rate $v$ arise from different dynamical processes to propagate the instability and, conse- quently, are characterized by different probability distributions. For small values of $v$, chain processes dominate the landslide dynamics: a few cells (a single cell in the limit of vanishing $v$ ) initially reach the instability threshold and, then, the event occurs as the effect of the relaxation processes that propagate the instability to neighbour cells. For very large values of $v$, an increasing number of cells initially reach instability and the domino effect is no more effective in determining the landslide event.

We found quite a good agreement between the synthetic pdf of the CA model and the landslide area pdf of three landslide inventories, and estimated the area of the cells of our grid, ranging from $35 \mathrm{~m}^{2}$ to $100 \mathrm{~m}^{2}$. From the comparison with the real data we realize that triggers of different origins can affect the stability of a slope with the same rate of approaching the instability. Therefore, it is crucial to get information about such a rate, rather than the nature of the triggering mechanism.

Finally, we propose a landslide-event magnitude scale related to $v$, which is a first attempt to relate the magnitude scale, $M_{L}$, to the strength of the perturbation acting on the system, instead of its visible effects. In such a classification of the landslide events, large values of the scale $M_{L}$ correspond to events with a fast approach to instability in a long distance of time, while small values of $M_{L}$ correspond to events with a slow approach to instability in a very close period of time. Conversely, from the approach of Malamud et al. (2004a) that predicts the same mean landslide area for all landslide events, from the analysis of the statistical properties of our CA model, we find that both the most likely event size and the mean event size are increasing functions of $M_{L}$.

Acknowledgements. The authors thank B. Malamud for providing the data of the three landslide inventories shown in Fig. 4. The authors are also very grateful to the Editor and the Reviewers for their valuable comments that significantly improved the manuscript. The authors acknowledge the support of the Research Project PRIN 2007: "Evaluation of geophysical and geological aspects of landslide susceptibility to severe natural events and relative land zoning" (2007LE8ZC5_003).

Edited by: B. Malamud

Reviewed by: O. Katz and two other anonymous referees

\section{References}

Christensen, K. and Olami, Z.: Scaling, phase transitions, and nonuniversality in a self-organized critical cellular-automaton model, Phys. Rev. A., 46, 1829-1838, 1992.

Dietrich, W. E., Reiss, R., Hsu, M. L., and Montgomery, D.: A process-based model for colluvial soil depth and shallow landslides using digital elevation data, Hydrol. Processes, 9, 383400, 1995.

Fredlund, D. G. and Rahardjo H.: Soil Mechanics for Unsatured Soils, Wiley-Interscience, New York, 1993. 
Guzzetti, F., Malamud, B., Turcotte, D., and Reichenbach, P.: Power-law correlations of landslides areas in central Italy, Earth Planet. Sci. Lett., 195, 169-183, 2002.

Hergarten, S. and Neugebauer, N.: Self-organized criticality in twovariable models, Phys. Rev. E., 61, 2382-2385, 2000.

Hergarten, S.: Landslides, sandpiles, and self-organized criticality, Nat. Hazards Earth Syst. Sci., 3, 505-514, 2003, http://www.nat-hazards-earth-syst-sci.net/3/505/2003/.

Iverson, M. R.: Landslide triggering by rain infiltration, Water Resour. Res., 26, 1897-1910, 2000.

Jensen, H. J.: Self-Organized Criticality: Emergent Complex Behavior, In: Physical and Biological Systems, Cambridge Univ. Press, Cambridge, UK, 1998.

Juanico, D. E., Longjas, A., Batac, R., and Monterola, C.: Avalanche statistics of driven granular slides in a miniature mound, Geophys. Res. Lett., 35, L19403, doi:10.1029/2008GL035567, 2008.

Katz, O. and Aharonov, E.: Landslides in a vibrating sand box: What controls types of slope failure and frequency magnitude relations?, Earth Planet. Sci. Lett., 247, 280-294, 2006.

Keefer, D. K.: Landslides caused by earthquakes, Bull. Geol. Soc. Am., 95, 406-421, 1984.

Malamud, B. D., Turcotte, D. L., Guzzetti, F., and Reichenbach, P.: Landslide inventories and their statistical properties, Earth Surf. Process. Landforms, 29, 687-711, 2004a.

Malamud, B. D., Turcotte, D. L., Guzzetti, F., and Reichenbach, P.: Landslides, earthquakes, and erosion, Earth Planet. Sci. Lett., 229, 45-59, 2004b.

Montgomery, D. R. and Dietrich, W. E.: A physically based model for the topographic control on shallow landsliding, Water Resour. Res., 30, 1153-1171, 1994.
Olami, Z., Feder, H. J. S., and Christensen K.: Self-organized criticality in a continuous, nonconservative cellular automaton modeling earthquakes, Phys. Rev. Lett., 68, 1244-1248, 1992.

Piegari, E., Cataudella, V., Di Maio, R., Milano, L., and Nicodemi, M.: A cellular automaton for the factor of safety field in landslides modelling, Geophys. Res. Lett., 33, L01403, doi:10.1029/2005GL024759, 2006a.

Piegari, E., Cataudella, V., Di Maio, R., Milano, L., and Nicodemi, M.: Finite driving rate and anisotropy effects in landslide modelling, Phys. Rev. E., 73, 026123-026129, 2006b.

Piegari, E., Cataudella, V., Di Maio, R., Milano, L., Nicodemi, M., and Soldovieri, M. G.: Electrical resistivity tomography and statistical analysis: a conceptual approach, J. Appl. Geophys., 68, 151-158, 2009.

Pietronero, L. and Schneider, W. R.: Fixed scale transformation approach to the nature of relaxation clusters in self-organized criticality, Phys. Rev. Lett., 66, 2336-2340, 1991.

Privman, V.: Finite size scaling and numerical simulation of statistical systems, World Scientific, Singapore, 1990.

Pruessner, G. and Jensen, H. J.: A solvable non-conservative model of self-organised criticality, Europhys. Lett., 58(2), 250-256, 2002.

Sidle, R. C.: A theoretical model of the effects of timber harvesting on slope stability, Water Resour. Res., 28, 1897-1910, 1992.

Stark, C. P. and Hovius, N.: The characterization of landslide size distributions, Geophys. Res. Lett., 28, 1091-1094, 2001.

Wu, W. and Sidle, R. C.: A distributed slope stability model for steep forested basins, Water Resour. Res., 31, 2097-2110, 1995. 